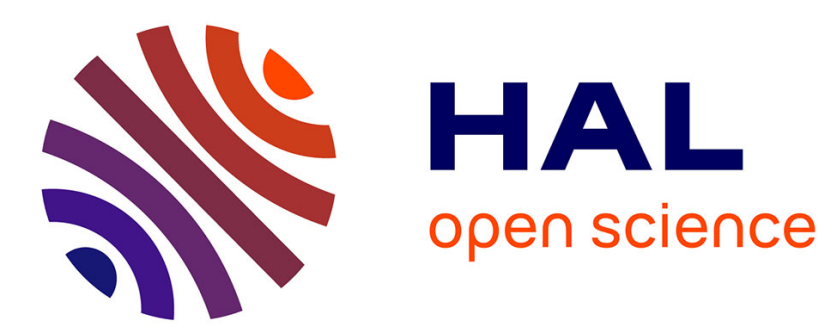

\title{
Inverse pickering emulsion stabilized by exfoliated hexagonal-boron nitride (h-BN)
}

Danae Gonzalez Ortiz, Céline Pochat-Bohatier, Julien Cambedouzou, Sébastien Balme, Mikhael Bechelany, Philippe Miele

\section{To cite this version:}

Danae Gonzalez Ortiz, Céline Pochat-Bohatier, Julien Cambedouzou, Sébastien Balme, Mikhael Bechelany, et al.. Inverse pickering emulsion stabilized by exfoliated hexagonal-boron nitride (h-BN). Langmuir, 2017, 33 (46), pp.13394-13400. 10.1021/acs.langmuir.7b03324 . hal-01675130

\section{HAL Id: hal-01675130 \\ https://hal.umontpellier.fr/hal-01675130}

Submitted on 10 Jun 2021

HAL is a multi-disciplinary open access archive for the deposit and dissemination of scientific research documents, whether they are published or not. The documents may come from teaching and research institutions in France or abroad, or from public or private research centers.
L'archive ouverte pluridisciplinaire HAL, est destinée au dépôt et à la diffusion de documents scientifiques de niveau recherche, publiés ou non, émanant des établissements d'enseignement et de recherche français ou étrangers, des laboratoires publics ou privés. 


\section{Inverse Pickering Emulsion Stabilized by Exfoliated}

\section{Hexagonal-Boron Nitride (h-BN)}

Danae Gonzalez Ortiz ${ }^{1}$, Céline Pochat-Bohatier ${ }^{1}$, Julien Cambedouzou ${ }^{2}$, Sébastien Balme ${ }^{1}$, Mikhael

Bechelany $^{1}$, Philippe Miele $e^{1,3} *$

${ }^{1}$ Institut Européen des Membranes, IEM UMR-5635, ENSCM, CNRS, Université de Montpellier, Place Eugene Bataillon, 34095, Montpellier, France

${ }^{2}$ Institut de Chimie Séparative de Marcoule, UMR 5257 CEA CNRS ENSCM Université de Montpellier,

BP17171, F-30207 Bagnols sur Ceze CEDEX, France

${ }^{3}$ Institut Universitaire de France (IUF)

*corresponding authors:

Mikhael Bechelany: mikhael.bechelany@umontpellier.fr, phone: +33467149167, Fax: +33467149119

Philippe Miele: philippe.miele@umontpellier.fr, phone: +33467149104 , Fax: +33467149119

\section{ABSTRACT}

The formation of inverse Pickering emulsions using exfoliated hexagonal boron nitride (h-BN) as an effective particulate stabilizer without using any surfactants is reported for the first time. The stability and the type of h-BN Pickering emulsions formulated with different $\mathrm{BN}$ concentrations and by varying oil/water $(\mathrm{o} / \mathrm{w})$ ratios are studied and discussed. First the emulsion structure is analyzed microscopically through optical and epifluorescence microscopies, and macroscopically by the study of the rheological behaviors. The average droplet size decreases with h-BN concentration whereas the emulsions achieve good stability at $2 \mathrm{wt} \% \mathrm{BN}$ concentrations and for a one to one o/w ratio. In all formulations, the emulsions are of water-in-oil (W/O) type due mainly to the hydrophobicity of h-BN. 
KEYWORDS. : Pickering emulsions, inverse emulsions, 2D materials, hexagonal boron nitride

Pickering emulsions or solid-stabilized emulsions were defined at the beginning of the $20^{\text {th }}$ century in pioneering works of Ramsden ${ }^{1}$ and Pickering. ${ }^{2}$ They are described as colloidal suspensions stabilized by solid nanoparticles instead of organic surfactants, which localize at the interface between two phases. As classical emulsions, they can be simply categorized based on the particle wettability: on the one hand, emulsions formed with hydrophilic particle normally stabilize Oil-in-Water $(\mathrm{O} / \mathrm{W})$ emulsions, or "direct" emulsions; on the other hand, those prepared with hydrophobic particles more probably form Water-in-Oil (W/O) emulsions. Sometimes W/O emulsions are also referred as "invert" or "reverse" emulsions. Unusual cases such as water-in-water emulsions, or double-emulsions, can also exist. ${ }^{3-4}$ Pickering emulsions have outstanding properties controlled by both formulation and process parameters, such as the kind of nanoparticles, the control of emulsion type and droplet size. These emulsions are good candidates to replace classical emulsions formulated with surfactants. Pickering emulsions offer specific features in life-science that are not shared with surfactant-based emulsions by avoiding the adverse effects of surfactants such as cytotoxicity. ${ }^{5}$ They can also build a barrier to diffusion of drug molecules and therefore behave as encapsulation systems. ${ }^{6}$ Coated emulsions, for example with hydroxyapatite, can play a role in targeting rules due to cell adhesion. ${ }^{7}$ Furthermore porous materials can be prepared using Pickering emulsion droplets as templates, in the same manner as hexagonal mesophases of surfactants act as templates for the manufacture of mesoporous inorganic materials such as MCM's. ${ }^{8}$ 
In the last years, many researchers have paid attention to the ability of two-dimensional (2D) materials to promote the formation of special class of Pickering emulsions. ${ }^{9}$ In this kind of emulsions, a large reduction of the free energy $(\Delta \mathrm{G})$ is found due to particles localized at the liquid-liquid interface, leading to the stabilization of the droplets. Graphene oxide (GO) is the most studied 2D material and has been considered to promote the formation of emulsions $^{10-11}$ due to its interesting property to act as a colloidal surfactant having a great interfacial activity at air-water, liquid-liquid, and liquid-solid interfaces. ${ }^{11-13,14} \mathrm{He}$ et al. investigated the effects of different conditions such as type of oil, the sonication time, the GO concentration, the oil/water ratio, and the $\mathrm{pH}$ value on the properties of the Pickering emulsions stabilized by GO. ${ }^{11}$ Creighton et al. presented the interfacial thermodynamics of 2D materials such as GO at liquid-liquid interfaces with applications in emulsification. ${ }^{9}$ Pickering emulsions stabilized by GO have been used as soft templates to design new functional hybrid materials. ${ }^{15,16,17}$ It should be pointed out that the graphene-based composites were usually synthesized through an "emulsion polymerization" since the graphene moieties can very well stabilize the monomer. Xie et al. reported the preparation of polystyrene (PS) particles via Pickering emulsion polymerization using GO as the stabilizer ${ }^{17}$ and Dao and co-workers prepared microspheres of poly(methyl methacrylate) (PMMA)/graphene composite with a core-shell structure by Pickering suspension polymerization. ${ }^{18}$

In this study, we focused our attention on h-BN as $2 \mathrm{D}$ material to prepare Pickering emulsions. Two-dimensional (2D) nanosheets, one-dimensional (1D) nanotubes, and zerodimensional (0D) fullerenes are examples of materials with reduced dimensionality. These low-dimensional structures exhibit new properties due to quantum confinement and/or surface and interfacial effects when compared to three-dimensional (3D) bulk substances. ${ }^{19}$ Similarly to carbon, BN can displays layered (hexagonal, h-BN), cubic (c-BN), and tubular structures 
(BN nanotubes). ${ }^{20}$ An hexagonal BN crystal structure with an exposed (002) surface have some advantages compared to graphite, such as very high thermal conductivity, mechanical strength and is electrically insulating (band gap of $\sim-6 \mathrm{eV}$ ). Furthermore, the thermal and chemical stability of $\mathrm{h}-\mathrm{BN}$ is better than that of graphite. ${ }^{21}$ Such outstanding properties are especially promising for fabrication of $\mathrm{BN}$-based functional materials with specific structure and performance. 2D hexagonal Boron nitride crystals, in the form of nanosheets (BNNS), are applied in the nanomedical field due to their good biocompatibility, and in scanning probe microscopy as ultrathin and stiff tips. ${ }^{22}$ They are also applied for filling polymeric or ceramic composites to strongly improve their thermal and mechanical performances. ${ }^{23-24}$

W/O Pickering emulsions are more suitable for some applications in comparison to direct emulsions due to their higher stability against collapsing. Frelichowska et al. used W/O emulsions for drug release and topic delivery using hydrophobic silica as stabilizer. ${ }^{25}$ Fei et al. synthesized modified graphene oxide/polyaniline nanocomposites from W/O emulsion through an interfacial polymerization mechanism. ${ }^{26} \mathrm{~A}$ patent application related to the preparation of $\mathrm{O} / \mathrm{W}$ and $\mathrm{W} / \mathrm{O}$ emulsions for cosmetic and dermatological applications has been reported using functionalized $\mathrm{BN}$ as stabilizer. In this procedure, surface $\mathrm{BN}$ was modified with siloxane polymer and inorganic pigments. ${ }^{27}$ While there is a big aim to develop a new method to prepare materials with specific shapes reducing the number of synthesis steps, there are also now important challenges concerning the direct preparation of W/O emulsions without functionalization of the nanoparticles.

One of the most important properties of emulsions for practical uses is their stability with time and when stress is applied which can be characterized from rheological measurements. The particles may form a transient network that reduces the mobility of the droplets, which leads to better stability. The flow behavior and the deformation of the emulsions stabilized by nanoparticles depend on different factors such as droplet size and morphology, dispersed 
phase volume fraction $(\phi)$, particles concentration and nature of interactions between the particles and the droplets in the continuous phase. ${ }^{[11]}$ Among these parameters, the particle concentration will influence the apparent viscosity by forming dispersed phase droplets of various size and droplets number with oil-water interface covered by solid particles.

In this work, the stability of W/O Pickering emulsions stabilized by exfoliated $\mathrm{h}-\mathrm{BN}$ and without further functionalization was evaluated for different concentrations and for various oil/water volume ratios. Ethyl benzoate was ascribed as the oil phase, because this aromatic organic solvent is almost completely insoluble in water and has been already successfully used for preparing Pickering emulsion with graphene oxide by He et al. ${ }^{11}$ The characteristics of Pickering emulsions will be discussed using a multiscale approach. First, the BN nanosheets were characterized by AFM and SAXS. Then the emulsion structure was analyzed microscopically through optical and epifluorescence microscopy, and macroscopically by rheological responses. The future perspectives with the proposed emulsion is to use them as templates to design new interesting materials with valuable properties, such as porous membranes or injectable scaffolds for drug delivery.

The h-BN stabilized emulsions were prepared by adding ethyl benzoate, in different o/w ratios, to aqueous suspensions of $\mathrm{BN}$ after a two-steps sonication procedure. The first step helps to exfoliate the $\mathrm{BN}$ with initial particle size of $3 \mu \mathrm{m}$ and thickness around $1 \mu \mathrm{m}$, the second step leads to the desired emulsion. Atomic force microscopy (AFM) was performed to confirm the BN exfoliation, leading to h-BN nanoparticles with a thickness around 2-6 nm and a lateral size in the range of 10 to $90 \mathrm{~nm}$ (Supporting Information, Figure S1). Based on AFM images and taking into account the width of one nanosheet $(0.36 \mathrm{~nm})$, we could assume our BNNS are generally composed of 6-16 layers. A SAXS analysis was performed on an emulsion prepared with $2 \mathrm{wt} \% \mathrm{BN}$ and an o/w ratio equal to 1 . The SAXS plot (Supporting information, Figure S2) of the emulsion displays a combination of the peaks characteristic of 
intermolecular distances of water and ethyl benzoate, and also a sharp peak at $\mathrm{q}=18.9 \mathrm{~nm}^{-1}$ corresponding to the $\mathrm{BN}$ inter-layer distance, giving the evidence of the presence of these three components.
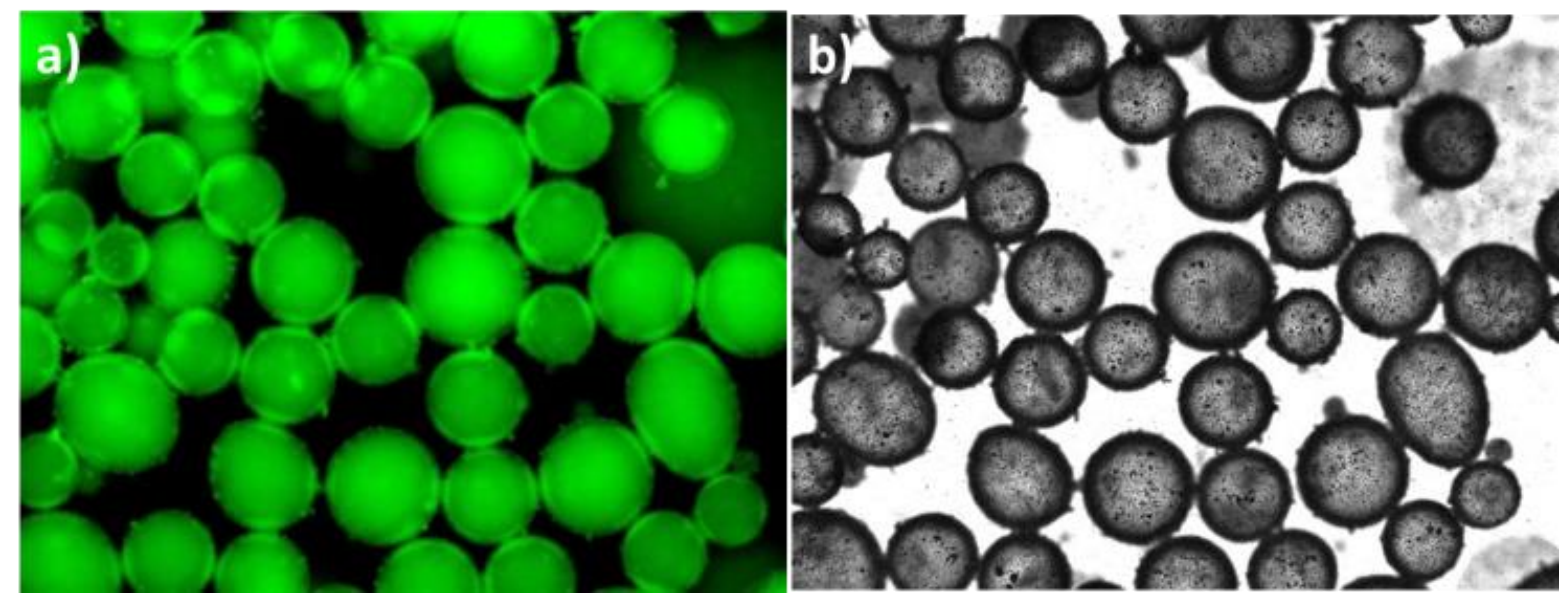

Figure 1. Epifluorescence images of Pickering emulsion BN 2wt\%_R1 a) Fluorescence and b) Differential interface contrast (DIC) techniques.

The type of obtained emulsion was identified by epifluorescence microscopy. Figure 1a shows labeled water inside the droplets stabilized by h-BN. The continuous phase outside droplets is thus assumed to be ethyl benzoate. These microphotographs clearly evidence that the obtained emulsions are of W/O type. Figure $1 \mathrm{~b}$ shows differential interface image contrast (DIC) images. A contrast difference is observed between the continuous and dispersed phase, namely oil and water. A black layer can be distinguished at the interface between oil and water which strongly suggests the presence of a shell of h-BN nanosheets around the droplets. 

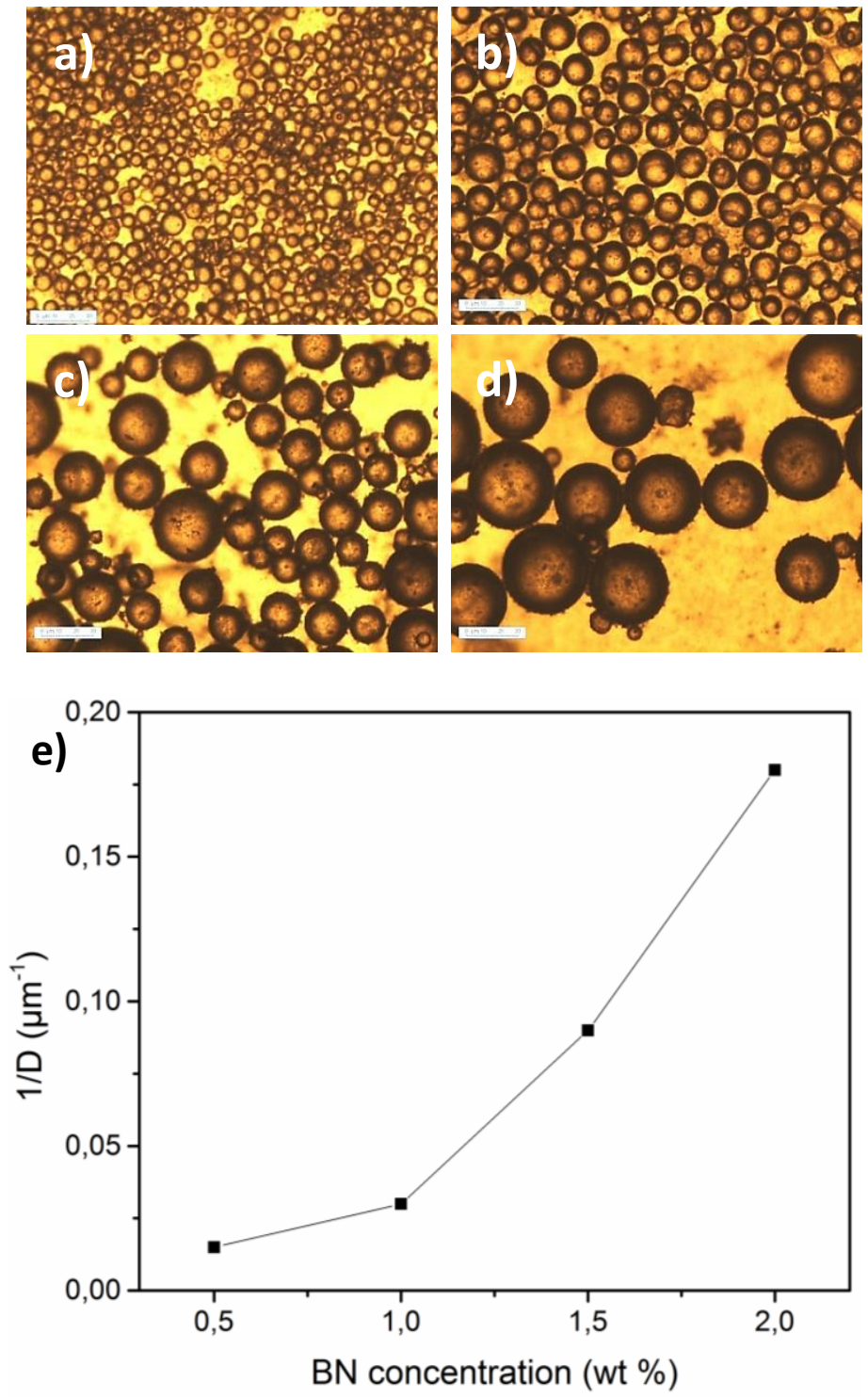

Figure 2. Optical microscopy images of h-BN emulsions at different concentrations: a) 2 wt $\%$ $\mathrm{BN}$, b) $1.5 \mathrm{wt} \% \mathrm{BN}, \mathrm{c}) 1.0 \mathrm{wt} \% \mathrm{BN}$ and d) $0.5 \mathrm{wt} \% \mathrm{BN}$ with a o/w ratio of 1 (The scale bars in the images correspond to $30 \mu \mathrm{m}$ length), e) Inverse of the average emulsion droplet size stabilized with h-BN particles at different concentrations $(0.5-2 \mathrm{wt} \%)$ at a fixed o/w ratio of 1 (measurements based on 50 droplets). 
The emulsion microstructure and drop size were analyzed by optical microscopy. Figure 2a-d shows microscopy images taken for the emulsions prepared with different h-BN particle concentrations, with ethyl benzoate/water volume ratio constant at 1 . The quantification of these results is shown in Figure 2e. The average droplet diameters range from $6 \mu \mathrm{m}$ at the highest $\mathrm{BN}$ concentration (2 wt\%) to $43 \mu \mathrm{m}$ at the lowest $\mathrm{BN}$ concentration $(0.5 \mathrm{wt} \%)$ when the o/w ratio is 1 . Thus, Pickering emulsions with different droplet sizes could be obtained by tuning the concentration of $\mathrm{BN}$ at constant amplitude settings $(55 \%)$. As expected, the average drop size of solid-stabilized emulsions decreases with increasing particle concentration, as more particles are available to stabilize small droplets. ${ }^{28}$ Decreasing the solid particle concentration led not only to a larger emulsion droplet but also to a greater heterogeneity in the droplets sizes and shapes, which signifies less efficient emulsion conditions. In our case, unlike other systems stabilized with spherical particles, the plot between the inverse of droplet size and the BN concentration (Figure 2e) displays a non linear dependence. This means that the emulsion is not limited by a coalescence phenomenon. Basically, the stability of a single particle at the interface is governed by the stabilization energy per particle. On the contrary than very small nanospheres (radius below 1-10 nm), it has been shown that the adsorption of graphene-based sheets never reaches a reversible regime due to graphene atomical thickness. ${ }^{29-30}$ In our system, the surface coverage ${ }^{31}$ was evaluated assuming that particle anchoring at the oil-water interface was always complete and irreversible. The resulting emulsions exhibit a drop diameter that is controlled by the mass of particles and their packing at the interface. Assuming that all particles are adsorbed at the oilwater interface, simple geometrical considerations show that the surface coverage is larger than 1 (Table S6), which evidences that the droplet are covered by multilayered particles or aggregates. ${ }^{32}$ The multilayer coverage was also reported in the case of GO, where it was estimated to stack up to 10 monolayers under certain conditions. ${ }^{33}$ From thermodynamics 
calculation, it was suggested that the 2D material multilayers at the interface effectively cover the droplet surface and inhibit collisions-induced coalescence or ripening. ${ }^{9}$ These emulsions stabilized with exfoliated h-BN are comparable in terms of stability and droplet size behaviors with other emulsions stabilized with 2D materials such as GO.

Preparing emulsions with h-BN has major interest due to the $\mathrm{BN}$ higher biocompatibility and its other unique properties, such as wide band gap, electrical-insulating properties, high thermal conductivity, high stability and resistance to oxidation, compared to other sheet-like materials, e.g. GO. Furthermore, numerous in vitro and in vivo assays have confirmed the toxicity of C-based materials. Alternatively $\mathrm{BN}$ possesses a better biocompatibility and a lower cytotoxicity. ${ }^{34-35,36}$

It is known that the droplet size is extremely sensitive to the amount of energy delivered to the biphasic system. This one has a direct relationship with the amplitude and intensity of the ultrasonic device. In order to decouple the influence of the energy delivered to the system with the amount of $\mathrm{BN}$ in the droplet size, emulsions formulated with $2 \mathrm{wt} \% \mathrm{BN}$ and an o/w ratio of 1 were first prepared using different amplitudes (20 to $80 \%$ ). Results shows (Table S3) that the emulsion droplet size do not change when different amplitudes are applied to the system. Then, the influence of the power delivered to the system by the same ultrasonic device on the droplet size was also evaluated. It has not big influence in the droplet size in the range 20 to $75 \mathrm{~W}$. For higher power, the mean droplet size is slightly smaller, but it is not really significant. So it can be conclude that the emulsifying process used in this study reached its limit to provide energy to the emulsion to produce smaller droplets with $2 \mathrm{wt} \%$ BN. This explains the gap with the linear dependency previously observed for lower BN load. Samples with different BN loads and same o/w ratio were then sonicated under constant 
power (40W) (Table S5). The obtained results confirm that the BN load is the main parameter affecting the droplet diameter with this ultrasonic probe used as emulsifying device.

The BN based-emulsions have been stored at ambient temperature for 14 days for studying their stability. We observed that emulsions formulated with $\mathrm{o} / \mathrm{w}$ ratios $\geq 0.6$ displayed two phases, one is the emulsified phase composed of 62 to $85 \%$ of volume fraction and the other is the oil phase resulting from the separation and sedimentation of the continuous phase due to its high density (mass density at $20^{\circ} \mathrm{C}$ is $1045 \mathrm{~kg} \mathrm{~m}^{-1}$ ). Above the random close packing (corresponding to a dispersed water volume fraction $\phi_{\mathrm{w}}>0.66$ ), the droplets can not move freely and they are trapped by the neighbors leading to emulsion destabilization after few hours preparation (Supporting information, Table S1). It was also observed that the emulsions with higher $\mathrm{BN}$ concentration have higher emulsion stability index (ESI), determined from the volume ratio of the emulsified phase after 14 days and emulsion after 3 hours. The emulsion with $2 \mathrm{wt} \% \mathrm{BN}$ and a o/w ratio of $1(\mathrm{BN} 2 \mathrm{wt} \%$ R 1$)$ is more stable along the time than the rest of emulsion, and shows an ESI around $94 \%$. In our system we observed that when the emulsion completely breaks down above the random close packing, there is a separation between two bulk phases: one very clear corresponds to the water phase and the other being whitish. Thereby we suggest that almost all particles are dispersed in the oil phase due the degree of hydrophobiciy of BN. A small part of BN particles could be adsorbed at the flat interface between oil and water.

Other phenomena was also observed by Binks et al. when emulsion stability is limited by coalescence phenomenon, destabilization occurs such as rapid film growth in hydrophilic tubes induced by the coalescence of spherical particles-stabilized emulsion drops with a flat oil-water interface separating the bulk oil and water phases. ${ }^{37}$ 
In our case, the emulsions are stabilized by multilayers of $2 \mathrm{D}$ material which inhibit the coalescence. Thus, we could propose two hypotheses to explain that emulsions are not stable above the close packing. The first one is based on that sheet-sheet interaction may promote multilayer film formation. In this case, the first layer packs imperfectly at the interface, leaving bare patches of liquid-liquid (L-L) interfaces that can interact with subsequent layers in a diffuse interfacial zone. For atomically thin plates, the second layer lies very close (within $1 \mathrm{~nm}$ ) to the L-L interfaces, and on this length scale, thermal molecular fluctuations allow the contact between the layer and both dispersed and continuous phases. In this way, the layers could contain interstitial spaces and then subsequent layers are too far from the interface to interact energetically with both liquid phases. There are two possible deposition pathways: First, the so-called "sequential close packing" route is characterized by an ordered layer-bylayer deposition. Second, a "random ballistic deposition" which has an irrespective prior coverage can take place. In the case of GO, it has been shown that at equal number of material layers, the stabilization energy is lower for the random deposition than close packing. This may explain the instability of emulsion. ${ }^{9}$

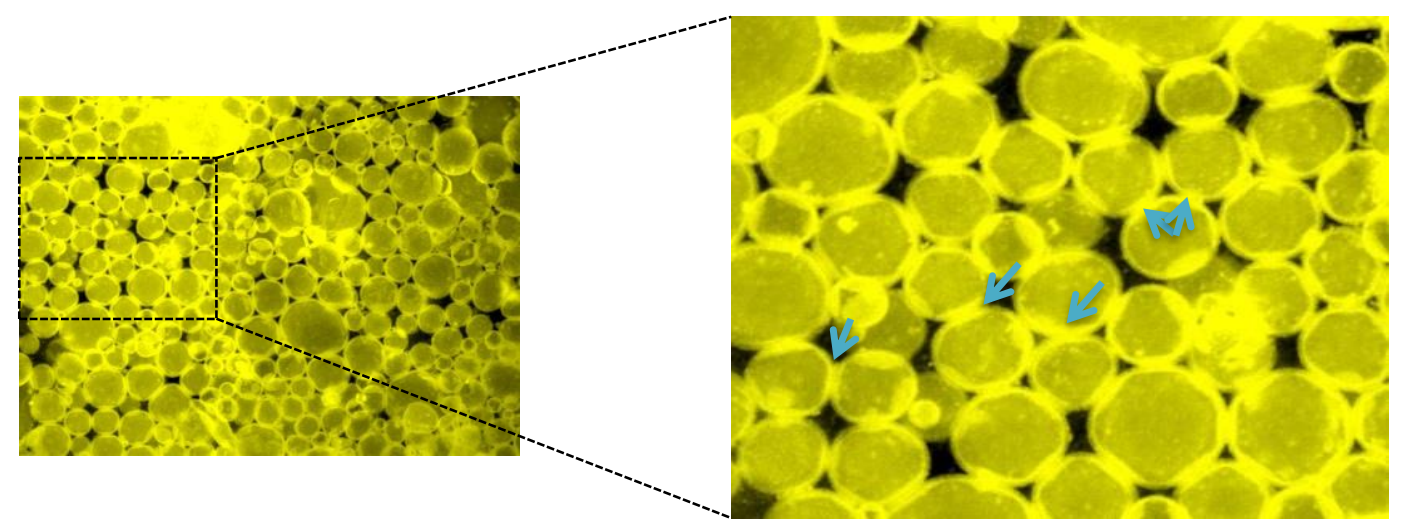

Figure 3. Epifluorescence images of Pickering emulsion $\mathrm{BN} 2 \mathrm{wt} \%$ R1 showing droplet deformation due to particle jamming. 
In the second hypothesis, we consider a stabilization mechanism called 'droplet bridging'. The emulsions prepared by ultrasonic probe display long-term stability when the dispersed phase volume fraction is well below the random close packing $(\varphi=0.65)$. Partially hydrophobic particles with contact angles somewhat greater than $90^{\circ}$ tend to stabilize waterin-oil emulsion where particles slightly protrude from droplets surface to maintain the equilibrium contact angle; this also facilitates efficient packing and steric barrier against coalescence. Particles concentration also plays an important role in the surface coverage. In fact, when coverage is very high, significant droplet deformation could be observed due to jamming of the particles at the droplet interfaces (Figure 3). Although the droplets were in close proximity, their interfaces were not joined because of the high particle coverage. This effect has been observed by Zhang et al. ${ }^{38}$ using layered double hydroxides particles for the preparation of Pickering emulsions. Moreover, droplets bridging has been observed by Frost et al. ${ }^{39}$ when ionic liquids are used for Pickering emulsions preparation. 


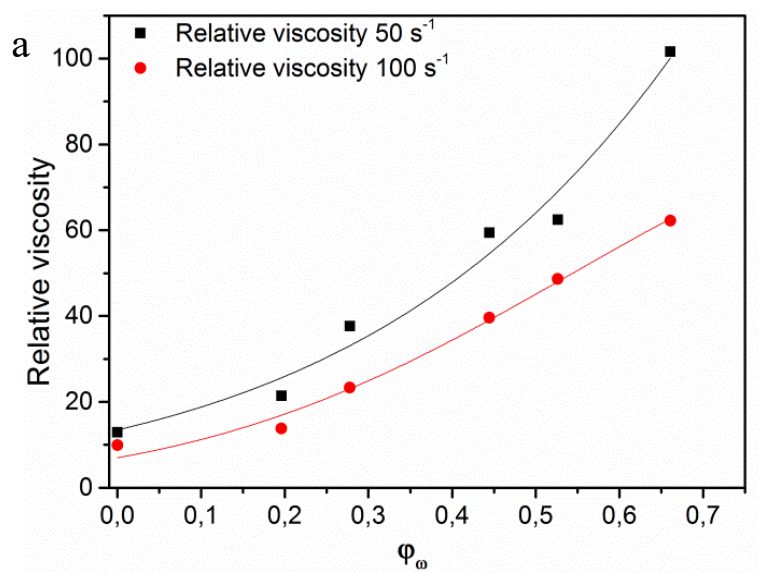

$\mathrm{b}$
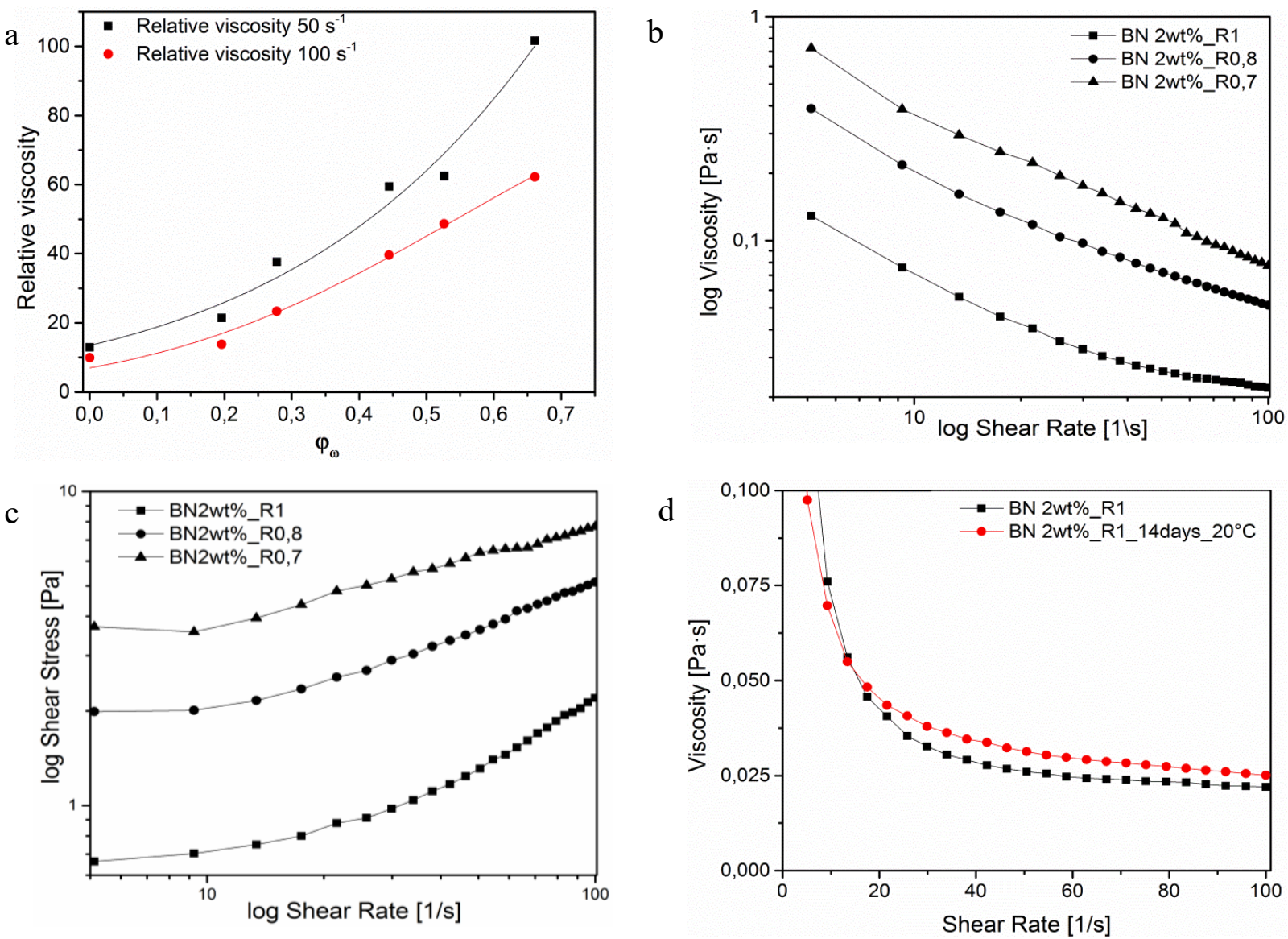

Figure 4. a) Relative viscosity curves in function of water volume fraction $\left(\phi_{\mathrm{w}}\right)$ for emulsions formulated at $2 \mathrm{wt} \% \mathrm{BN}$ concentration at shear rate $50 \mathrm{~s}^{-1}$ and $100 \mathrm{~s}^{-1}$ (Lines are eye-guides). For values of $\phi_{\mathrm{w}}$ up to 0.66 the emulsions broke down. b) Viscosity curves of emulsion with 2 wt $\%$ h-BN concentration and different $\mathrm{o} / \mathrm{w}$ ratios $(\mathrm{R}=0.7$ to 1$)$; c) Flow curves of emulsion with $2 \mathrm{wt} \%$ h-BN concentration and different o/w ratios ( $\mathrm{R}=0.7$ to 1$) \mathrm{d}$ ) Viscosity curves of freshly prepared BN2 wt_R1 emulsion, and after 14 days.

By rheological measurements, it is also possible to study the emulsion stability. For that purpose, the effect of varying the water volume fraction $\left(\phi_{\mathrm{w}}\right)$ was investigated by concentrating the emulsions at constant particle concentration using a rotational rheometry 
technique. Figure 4a shows the relative viscosity (ratio between the apparent viscosity of the emulsion and the oil phase viscosity) as a function of the water volume fraction. Our system behaves as the literature predicts, increasing the viscosity when rising the $\phi_{\mathrm{w}}$. It is observed that increasing $\phi_{\mathrm{w}}$ increases the relative viscosity from 12.9 , corresponding to low volume fraction $(<0.05)$, to 101.6 for high volume fraction $(0.66)$ when the shear rate is $50 \mathrm{~s}^{-1}$. The same behavior was found when the shear rate was $100 \mathrm{~s}^{-1}$, the relative viscosity increasing from $9.9\left(\phi_{\mathrm{w}}=0.05\right)$ to $62.3\left(\phi_{\mathrm{w}}=0.66\right)$. This fact may be due to the increase of water content and to the possibility that droplets tend to pack closer, adding a significant contribution to the emulsion viscosity. We observed also that w/o emulsions stabilized with exfoliated h-BN formulated with $\phi_{\mathrm{w}}$ above 0.66 and $2 \mathrm{wt} \% \mathrm{BN}$ concentration are not stable anymore after preparation or after a few hours and broke down due to the fact that maximum random close packing has been reached for our system. ${ }^{38,40}$ The present results thus show that the maximum packing fraction for w/o emulsion made with ethyl benzoate and $\mathrm{BN}$ is close to 0.64 , as described in the literature for monodisperse spherical drops. The aim of the study is to use this emulsion as a potential soft template for the preparation of new porous materials. In this case, the inner phase will give the porosity volume and the continuous phase will form the material matrix. If the continuous phase volume is too limited, it will give weak mechanical properties to the solid material. However, it should be noted that the maximum total porous volume reached with the present emulsion to make for instance a membrane would be ca. $64 \%$. The preparation of porous nanocomposites materials from medium internal phase emulsions has been already reported in the literature. Pickering emulsions containing only $60 \%$ of disperse phase could lead to open porous polymer foams with low densities and high degrees of pore interconnectivity. ${ }^{41}$ 
The rheological behavior of pure ethyl benzoate, pure water, and of the obtained emulsions was analyzed by measuring the viscosity in function of the shear rate at $20{ }^{\circ} \mathrm{C}$. The measurement was carried out within the 10 min following emulsification where the emulsions are stable, the duration of experiment was around 4 min and no phase separation was observed after the analysis. Figure $4 \mathrm{~b}$ shows the viscosity curves obtained for the $\mathrm{BN}$ emulsions formulated at $2 \mathrm{wt} \%$ of $\mathrm{BN}$ and different $\mathrm{O} / \mathrm{W}$ ratios. In this study, standard coneand-plate geometry with $50 \mathrm{~mm}$ diameter and a $1^{\circ}$ cone angle was used. The maximal acceptable droplet diameter for this type of geometry is defined by this equation:

$\mathrm{d} \leq \mathrm{a} / 10$

The gap set at the center of the cone (a) has a value of $101 \mu \mathrm{m}$ so the diameter of droplet must be below $10 \mu \mathrm{m}$, and our droplets meet this condition with a $6 \mu \mathrm{m}$ diameter. At any given shear rate, the emulsions have a higher viscosity than pure phases (Figure 3b), especially at low shear rates due to the even dispersion of h-BN in the water and oil mixture, acting as a stabilizer. The emulsions display a typical shear-thinning flow behavior, characterized by decreasing the viscosity as the applied shear rate increases, approaching to a constant value at high shear rates. At low shear rates, the measured emulsion viscosity is higher, indicating that at rest the droplet close-packing is responsible of viscosity. When a higher shear rate is applied, the droplets start to be organized into layers or strings, and the rheogram displays a decrease in viscosity. The measurements were performed three times using the same emulsion to ensure the repeatability of the test. The results show (Supporting information, Table S2) that the test could be repeated without changes in viscosity data. Figure $\mathbf{4 c}$ shows the curve of shear stress as a function of the shear rate of an emulsion containing $2 \mathrm{wt} \%$ of $\mathrm{h}-\mathrm{BN}$ and different $\mathrm{O} / \mathrm{W}$ ratios $(\mathrm{R}=0.7$ to 1$)$. The flow curves exhibit a yield stress dependent on the $\mathrm{o} / \mathrm{W}$ ratio. The reduction of the $\mathrm{o} / \mathrm{w}$ ratio from 1 to 0.7 corresponds also to an increase of the inner phase volume $(+43 \% \mathrm{v} / \mathrm{v}$ of water) and thus to a concentration of the emulsion with a higher 
amount of dispersed phase. As a consequence, an increase of the viscosity and of the yield stress is observed. In Figure 4d, the viscosity curves of the emulsion containing 2 wt $\%$ BN and with an o/w ratio of 1 are shown as freshly prepared and after 14 days. No change in the apparent viscosity is observed after 14 days.

In summary, non-modified 2D BN material has been used for the first time as stabilizing agent for the preparation of inverse Pickering emulsions. The epifluorescence images revealed that the emulsions are of water-in-oil type. Microscopy images showed that the average droplet size decreases with increasing the BN concentration. The emulsions possess good stability for $2 \mathrm{wt} \% \mathrm{BN}$ concentrations and oil/water ratio equal to 1 . The kinetic stability showed that the emulsions prepared with $2 \mathrm{wt} \% \mathrm{BN}$ are the most stable, and rheology tests confirmed this result, showing that no change on the stability occurred after 14 days. In addition, rheological measurements performed on the emulsions with $2 \mathrm{wt} \%$ BN exhibited higher viscosity for water volume fraction close to 0.64 . This value corresponds to the maximum packing volume fraction of water in ethyl benzoate, for drops of spherical shape stabilized by h-BN nanosheets. These emulsions may be good candidates to design new interesting materials with valuable properties, such as porous membranes. In addition, emulsions formulated with h-BN are particularly interesting due to their high biocompatibility compared with other 2D materials such as GO, and their potential application in biofields, taking into account the fact that inverse emulsions possess higher stability than classical emulsions.

ACKNOWLEDGEMENTS: The work was financially supported by the French National Agency (ANR) through the LabEx CheMISyst (ANR-10-LABX-05-01) and the IUF project of Prof. P. Miele. 
SUPPORTING INFORMATION. An experimental section, additional AFM and SAXS analyses and a stability Table are supplied as Supporting Information.

\section{REFERENCES}

1. Ramsden, W., Separation of Solids in the Surface-Layers of Solutions and'Suspensions'(Observations on Surface-Membranes, Bubbles, Emulsions, and Mechanical Coagulation).--Preliminary Account. Proceedings of the royal Society of London 1903, 72, 156-164. https://doi.org/10.1098/rspl.1903.0034

2. Pickering, S. U., CXCVI.- - emulsions. Journal of the Chemical Society, Transactions 1907, 91, 2001-2021. https://doi.org/10.1039/ct9079102001

3. Capron, I.; Costeux, S.; Djabourov, M., Water in water emulsions: phase separation and rheology of biopolymer solutions. Rheologica Acta 2001, 40 (5), 441-456. https://doi.org/10.1007/s003970100161

4. $\quad$ Nguyen, B. T.; Wang, W.; Saunders, B. R.; Benyahia, L.; Nicolai, T., pH-responsive water-in-water Pickering emulsions. Langmuir 2015, 31 (12), 3605-3611. https://doi.org/10.1021/la5049024

5. Marto, J.; Gouveia, L.; Gonçalves, L.; Chiari-Andréo, B.; Isaac, V.; Pinto, P.; Oliveira, E.; Almeida, A.; Ribeiro, H., Design of novel starch-based Pickering emulsions as platforms for skin photoprotection. Journal of Photochemistry and Photobiology B: Biology 2016, 162, 56-64. https://doi.org/10.1016/j.jphotobiol.2016.06.026

6. Simovic, S.; Hui, H.; Song, Y.; Davey, A. K.; Rades, T.; Prestidge, C. A., An oral delivery system for indomethicin engineered from cationic lipid emulsions and silica nanoparticles. Journal of Controlled Release 2010, 143 (3), 367-373. https://doi.org/10.1016/i.jconrel.2010.01.008

7. Maeda, H.; Okada, M.; Fujii, S.; Nakamura, Y.; Furuzono, T., Pickering-type waterin-oil-in-water multiple emulsions toward multihollow nanocomposite microspheres. Langmuir 2010, 26 (17), 13727-13731. https://doi.org/10.1021/la102529d

8. Kresge, C.; Leonowicz, M.; Roth, W.; Vartuli, J.; Beck, J., Ordered mesoporous molecular sieves synthesized by a liquid-crystal template mechanism. Nature 1992, 359 (6397), 710-712. https://doi.org/10.1038/359710a0

9. Creighton, M. A.; Ohata, Y.; Miyawaki, J.; Bose, A.; Hurt, R. H., Two-dimensional materials as emulsion stabilizers: interfacial thermodynamics and molecular barrier properties. Langmuir 2014, 30 (13), 3687-3696. https://doi.org/10.1021/la500216n

10. Kim, J.; Cote, L. J.; Huang, J., Two dimensional soft material: new faces of graphene oxide. Accounts of Chemical Research 2012, 45 (8), 1356-1364. https://doi.org/10.1021/ar300047s

11. He, Y.; Wu, F.; Sun, X.; Li, R.; Guo, Y.; Li, C.; Zhang, L.; Xing, F.; Wang, W.; Gao, J., Factors that affect pickering emulsions stabilized by graphene oxide. ACS applied materials \& interfaces 2013, 5 (11), 4843-4855. https://doi.org/10.1021/am400582n 
12. Sun, Z.; Feng, T.; Russell, T. P., Assembly of graphene oxide at water/oil interfaces: tessellated nanotiles. Langmuir 2013, $29 \quad$ (44), 13407-13413. https://doi.org/10.1021/la402436w

13. Texter, J., Graphene oxide and graphene flakes as stabilizers and dispersing aids. Current Opinion in Colloid \& Interface Science 2015, 20 (5), 454-464. https://doi.org/10.1016/j.cocis.2015.11.006

14. Kim, J.; Cote, L. J.; Kim, F.; Yuan, W.; Shull, K. R.; Huang, J., Graphene oxide sheets at interfaces. Journal of the American Chemical Society 2010, 132 (23), 8180-8186. https://doi.org/10.1021/ja102777p

15. Tang, M.; Wang, X.; Wu, F.; Liu, Y.; Zhang, S.; Pang, X.; Li, X.; Qiu, H., Au nanoparticle/graphene oxide hybrids as stabilizers for Pickering emulsions and $\mathrm{Au}$ nanoparticle/graphene oxide@ polystyrene microspheres. Carbon 2014, 71, 238-248. https://doi.org/10.1016/i.carbon.2014.01.034

16. Moghaddam, S. Z.; Sabury, S.; Sharif, F., Dispersion of rGO in polymeric matrices by thermodynamically favorable self-assembly of $\mathrm{GO}$ at oil-water interfaces. RSC Advances 2014, 4 (17), 8711-8719. https://doi.org/10.1039/c3ra46342g

17. Xie, P.; Ge, X.; Fang, B.; Li, Z.; Liang, Y.; Yang, C., Pickering emulsion polymerization of graphene oxide-stabilized styrene. Colloid \& Polymer Science 2013, 291 (7), 1631. https://doi.org/10.1007/s00396-013-2897-x

18. Dao, T. D.; Erdenedelger, G.; Jeong, H. M., Water-dispersible graphene designed as a Pickering stabilizer for the suspension polymerization of poly (methyl methacrylate)/graphene core-shell microsphere exhibiting ultra-low percolation threshold of electrical conductivity. Polymer 2014, 55 (18), 4709-4719. https://doi.org/10.1016/i.polymer.2014.07.038

19. Pakdel, A.; Zhi, C.; Bando, Y.; Golberg, D., Low-dimensional boron nitride nanomaterials. Materials Today 2012, 15 (6), 256-265. https://doi.org/10.1016/S13697021(12)70116-5

20. Zhi, C.; Bando, Y.; Tang, C.; Kuwahara, H.; Golberg, D., Large-scale fabrication of boron nitride nanosheets and their utilization in polymeric composites with improved thermal and mechanical properties. Advanced Materials 2009, 21 (28), 2889-2893. https://doi.org/10.1002/adma.200900323

21. Golberg, D.; Bando, Y.; Huang, Y.; Terao, T.; Mitome, M.; Tang, C.; Zhi, C., Boron nitride nanotubes and nanosheets. ACS Nano 2010, 4 (6), 2979-2993. https://doi.org/10.1021/nn1006495

22. Ciofani, G.; Genchi, G. G.; Liakos, I.; Athanassiou, A.; Dinucci, D.; Chiellini, F.; Mattoli, V., A simple approach to covalent functionalization of boron nitride nanotubes. Journal of Colloid and Interface Science 2012, 374 (1), 308-314. https://doi.org/10.1016/i.jcis.2012.01.049

23. Wang, X.; Pakdel, A.; Zhang, J.; Weng, Q.; Zhai, T.; Zhi, C.; Golberg, D.; Bando, Y., Large-surface-area BN nanosheets and their utilization in polymeric composites with improved thermal and dielectric properties. Nanoscale Research Letters 2012, 7 (1), 1-7. https://doi.org/10.1186/1556-276X-7-662

24. Biscarat, J.; Bechelany, M.; Pochat-Bohatier, C.; Miele, P., Graphene-like BN/gelatin nanobiocomposites for gas barrier applications. Nanoscale 2015, 7 (2), 613-618. https://doi.org/10.1039/c4nr05268d

25. Frelichowska, J.; Bolzinger, M.-A.; Pelletier, J.; Valour, J.-P.; Chevalier, Y., Topical delivery of lipophilic drugs from $\mathrm{o} / \mathrm{w}$ Pickering emulsions. International Journal of Pharmaceutics 2009, 371 (1), 56-63. https://doi.org/10.1016/i.ijpharm.2008.12.017 
26. Fei, X.; Xia, L.; Chen, M.; Wei, W.; Luo, J.; Liu, X., Preparation and Application of Water-in-Oil Emulsions Stabilized by Modified Graphene Oxide. Materials 2016, 9 (9), 731. https://doi.org/10.3390/ma9090731

27. Gers-Barlag, H.; Müller, A., Finely dispersed emulsifier-free systems of the oil-inwater and water-in-oil type. Google Patents: 2003.

28. Binks, B.; Lumsdon, S., Pickering emulsions stabilized by monodisperse latex particles: effects of particle size. Langmuir 2001, 17 (15), 4540-4547. https://doi.org/10.1021/la0103822

29. Cote, L. J.; Kim, F.; Huang, J., Langmuir- Blodgett assembly of graphite oxide single layers. Journal of the American Chemical Society 2008, 131 (3), 1043-1049. https://doi.org/10.1021/ja806262m

30. Imperiali, L.; Liao, K.-H.; Clasen, C.; Fransaer, J.; Macosko, C. W.; Vermant, J., Interfacial rheology and structure of tiled graphene oxide sheets. Langmuir 2012, 28 (21), 7990-8000. https://doi.org/10.1021/la300597n

31. Lou, F.; Ye, L.; Kong, M.; Yang, Q.; Li, G.; Huang, Y., Pickering emulsions stabilized by shape-controlled silica microrods. RSC Advances 2016, 6 (29), 24195-24202. https://doi.org/10.1039/c6ra00360e

32. Destribats, M.; Gineste, S. p.; Laurichesse, E.; Tanner, H.; Leal-Calderon, F.; Héroguez, V. r.; Schmitt, V. r., Pickering emulsions: what are the main parameters determining the emulsion type and interfacial properties? Langmuir 2014, 30 (31), 93139326. https://doi.org/10.1021/la501299u

33. Imperiali, L.; Clasen, C.; Fransaer, J.; Macosko, C. W.; Vermant, J., A simple route towards graphene oxide frameworks. Materials Horizons 2014, 1 (1), 139-145. https://doi.org/10.1039/c3mh00047h

34. Lewinski, N.; Colvin, V.; Drezek, R., Cytotoxicity of Nanoparticles. Small 2008, 4 (1), 26-49. https://doi.org/10.1002/smll.200700595

35. Mansukhani, N. D.; Guiney, L. M.; Kim, P. J.; Zhao, Y.; Alducin, D.; Ponce, A.; Larios, E.; Yacaman, M. J.; Hersam, M. C., High-Concentration Aqueous Dispersions of Nanoscale 2D Materials Using Nonionic, Biocompatible Block Copolymers. Small 2016, 12 (3), 294-300. https://doi.org/10.1002/smll.201503082

36. Wang, K.; Ruan, J.; Song, H.; Zhang, J.; Wo, Y.; Guo, S.; Cui, D., Biocompatibility of graphene oxide. Nanoscale Research Letters 2010, 6 (1), 8. https://doi.org/10.1007/s11671$\underline{010-9751-6}$

37. Binks, B.; Clint, J.; Fletcher, P.; Lees, T.; Taylor, P., Growth of gold nanoparticle films driven by the coalescence of particle-stabilized emulsion drops. Langmuir 2006, 22 (9), 4100-4103. https://doi.org/10.1021/la052752i

38. Zhang, N.; Zhang, L.; Sun, D., Influence of emulsification process on the properties of Pickering emulsions stabilized by layered double hydroxide particles. Langmuir 2015, 31 (16), 4619-4626. https://doi.org/10.1021/la505003w

39. Frost, D. S.; Schoepf, J. J.; Nofen, E. M.; Dai, L. L., Understanding droplet bridging in ionic liquid-based Pickering emulsions. Journal of colloid and interface science 2012, 383 (1), 103-109. https://doi.org/10.1016/i.jcis.2012.06.010

40. Udoetok, I. A.; Wilson, L. D.; Headley, J. V., Stabilization of pickering emulsions by iron oxide nano-particles. Advanced Material Science 2016, 1 (1), 24-33. https://doi.org/10.15761/ams.1000107

41. Menner, A.; Verdejo, R.; Shaffer, M.; Bismarck, A., Particle-stabilized surfactant-free medium internal phase emulsions as templates for porous nanocomposite materials: polypickering-foams. Langmuir 2007, 23 (5), 2398-2403. https://doi.org/10.1021/la062712u 
TABLE OF CONTENTS GRAPHIC
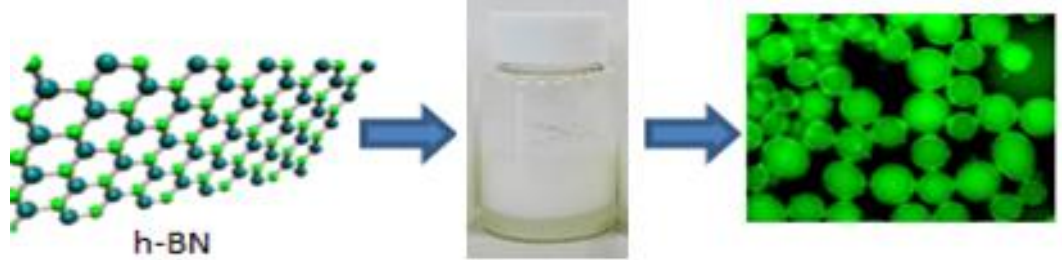